\title{
Evaluación del sistema de generación y distribución de vapor de una empresa cárnica
}

\author{
Evaluation of the steam generation and distribution system at a meat company
}

\author{
Y. Reyes-Betancourt iD ; R. González-de-la-Cruz ; Y. B. Rodríguez-Guerra iD ;. Liaño- \\ Abascal iD ; A. Pérez-Sánchez iD \\ DOI: https://doi.org/10.22517/23447214.23341 \\ Artículo de investigación científica y tecnológica
}

\begin{abstract}
Resumen- Este trabajo se realizó en el Combinado Cárnico “César Escalante Dellundé”, de la provincia de Camagüey, Cuba con el objetivo de evaluar su sistema de generación y distribución de vapor. Se obtuvo una eficiencia energética de la caldera pirotubular de $87,41 \%$, las pérdidas de calor al ambiente de las tuberías no aisladas ascendieron a $85764,31 \mathrm{~W}$, mientras que la dureza total del agua de alimentación a la caldera se encuentra 10,26 veces por encima de lo establecido por norma. Se propusieron dos alternativas de mejoras, consistentes en: 1) aislamiento de las tuberías por donde circula vapor de agua con el aislante Lana Mineral, y 2) precalentamiento del agua de alimentación a la caldera hasta $70{ }^{\circ} \mathrm{C}$. La aplicación de ambas propuestas de mejoras traerá consigo un ahorro de combustible de $23,56 \mathrm{~kg} / \mathrm{h}$, lo cual permite que se dejen de emitir $576 \mathrm{~m}^{3} / \mathrm{h}$ de gases de combustión al ambiente, además de un posibilitar un incremento de la eficiencia energética de la caldera en un $0,10 \%$. El costo total de inversión necesario para implementar las dos alternativas de mejoras propuestas alcanzó un valor de \$ 8117,35 pesos cubanos (PC), el cual se recupera en 2,36 meses considerando los ahorros económicos por año que se obtienen, los cuales ascienden a PC \$ 41 193,33 a causa de combustible que no se consume en la caldera.
\end{abstract}

Palabras claves - Caldera pirotubular, dureza total, eficiencia energética, emisiones, pérdidas de calor.

Absract - This work was carried out in the "César Escalante Dellundé" Meat Company, in the province of Camagüey, Cuba, with the objective of evaluating its steam generation and distribution system. An energy efficiency of the fire-tube boiler of $87.41 \%$ was obtained, the heat losses to the environment of the non-insulated pipes amounted to $85,764.31 \mathrm{~W}$, while the total hardness of the feed water to the boiler is $\mathbf{1 0 . 2 6}$ times above the

This manuscript was sent on February 10, 2020 and accepted on February 05, 2021.

Y. Reyes-Betancourt, especialista de Transportación Ferroviaria de la Empresa de Ferrocarriles Centro-Este, calle José Francisco Agüero, No. 301, Camagüey, Cuba. (email: yasnelirb@nauta.cu).

R. González-de la Cruz, Profesor Auxiliar Consultante de la Facultad de Ciencias Aplicadas de la Universidad de Camagüey, Carretera Circunvalación Norte, km 5 1⁄2, Camagüey, Cuba. (email: raul.gonzalez@reduc.edu.cu). standard value. Two alternatives for improvements were proposed, consisting of: 1) insulation of the pipes through which water vapor circulates with Mineral Wool, and 2) preheating the boiler feed water up to $70{ }^{\circ} \mathrm{C}$. The application of both proposals of improvements will result in fuel savings of $23.56 \mathrm{~kg} / \mathrm{h}$, which allows stop releasing about $576 \mathrm{~m}^{3} / \mathrm{h}$ of combustion gases to the environment, along with an increase of the energy efficiency of the boiler of $0.10 \%$. The total investment cost necessary to implement the two proposed improvement alternatives reached a value of $\$ 8$ 117,35 Cuban pesos (CUP), which is recovered in 2.36 months considering the economic savings per year obtained, which amount to CUP \$ 41193.33 due to fuel not consumed in the boiler.

Index Terms - Emissions, energetic efficiency, fire-tube boiler, heat losses, total hardness.

\section{INTRODUCCIÓN}

L ahorro de los recursos energéticos es un concepto - 1 importante en cualquier industria o planta química. El nivel de eficiencia del trabajo de las instalaciones térmicas constituye una parte fundamental en el ahorro o gasto de los mismos, especialmente, con respecto al combustible. Esto es aplicable al caso de las unidades generadoras de vapor, tal y como sucede con la caldera pirotubular, donde la eficiencia del sistema termoenergético depende en gran medida de las pérdidas asociadas a ellas [1].

Las calderas o generadores de vapor son un elemento fundamental en el funcionamiento de instalaciones industriales y de servicios. Constituyen el corazón de los sistemas termoenergéticos en los cuales están presentes ya que producen el vapor que entrega la energía térmica demandada por los procesos de calentamiento, esterilización, pasteurización etc., y la producción de potencia mecánica.

Y. B. Rodríguez-Guerra, Especialista de Producción del Combinado Cárnico de Camagüey, Carretera Circunvalación Norte, km 1 1/2, Camagüey, Cuba. (email: yilenabrg@nauta.cu).

N. Liaño-Abascal, Profesor Asistente de la Facultad de Ciencias Aplicadas de la Universidad de Camagüey, Carretera Circunvalación Norte, km 5 1/2, Camagüey, Cuba. (email: norlem.liano@reduc.edu.cu).

A. Pérez-Sánchez. Profesor Instructor de la Facultad de Ciencias Aplicadas de la Universidad de Camagüey, Carretera Circunvalación Norte, km 5 1/2, Camagüey, Cuba. (email: amauryps@nauta.cu). 
En las calderas de vapor pirotubulares los gases, producto de la combustión, circulan por el interior de los tubos, y en la mayoría de los casos la propia combustión se produce dentro de un tubo de mayor diámetro, de ahí su nombre (tubos de fuego), los cuales se encuentran sumergidos en la masa de agua. Todo el conjunto se encuentra rodeado por una envoltura o casco exterior rígido [2].

Los generadores de vapor pirotubulares de baja y media presión encuentran un uso extensivo en la industria química actual, siendo utilizados como fuente de tanto calor como vapor. Las calderas de baja presión son aquellas que operan entre 15 $20 \mathrm{kgf} / \mathrm{cm}^{2}$ de presión $\left(\sim 300 \mathrm{lb} / \mathrm{pulg}^{2}\right)$. Por encima de esta presión de trabajo, pero por debajo de las $64 \mathrm{kgf} / \mathrm{cm}^{2}(\sim 900$ $\mathrm{lb} / \mathrm{pulg}^{2}$ ) se encuentra el grupo de las calderas de media presión [3].

La eficiencia térmica es el indicador más importante del trabajo de un generador de vapor, ya que caracteriza el grado de aprovechamiento de la energía suministrada, o sea, la parte de esa energía que ha sido transferida al agente de trabajo. La eficiencia de la caldera se expresa en porcentaje o por término de evaporación, que indica la proporción de vapor generado por unidad de combustible quemado en el horno. Las mayores pérdidas que se producen en una caldera pirotubular, y que por tanto, afectan en mayor medida la eficiencia de las mismas, están determinadas por el calor sensible que se escapa con los gases de la combustión, determinado esto por la alta temperatura que llevan los mismos, y las pérdidas por radiación, presentes en todo momento, ya sea durante el funcionamiento o en los períodos de paradas por bajo consumo de vapor en los equipos instalados en la industria [1].

Varios autores han estudiado la eficiencia energética o térmica de calderas. En este sentido [4] desarrollaron un modelo matemático determinístico de procesamiento de registros experimentales, aplicable a un sistema generador de vaporprecalentador de aire en estado estacionario, operando con uno o dos combustibles simultáneamente para determinar la eficiencia térmica del mismo y la eficiencia con la que se oxida el combustible, así como también el rendimiento del intercambiador de calor. También [5] analizaron el comportamiento de los principales parámetros operacionales de una caldera pirotubular para obtener su eficiencia por el método directo e indirecto bajo condiciones de cargas reducidas, determinando igualmente el rendimiento energético y exergético del sistema y analizando las pérdidas que existen en la red de distribución debido a la falta de aislamiento. Por su parte [6] exponen un método para la evaluación de la eficiencia energética y el impacto ambiental de un generador pirotubular, describiendo las pérdidas que se producen en la caldera y los valores esperados de las mismas. Además, [7] presentaron una metodología para determinar la eficiencia energética y exergética de calderas pirotubulares de baja potencia, para posteriormente realizar un análisis de la influencia de algunos parámetros del proceso, tales como la temperatura de los gases de combustión en el valor a obtener de la eficiencia energética. Por otro lado, [8] efectuó un estudio energético del sistema de generación de vapor de una planta de fabricación de alimentos donde se utiliza una caldera de $125 \mathrm{hp}$ de la línea Cleaver Brooks, en donde se elaboró también una propuesta para el mejoramiento de la eficiencia de operación de la misma, empleando para ello una metodología lógica y fundamentada en balances de masa y energía para determinar la eficiencia de combustión y la eficiencia de operación de la caldera. Por último, [9] calcularon la eficiencia energética de una caldera pirotubular por el método indirecto a partir de la evaluación de las diferentes perdidas de calor asociadas al proceso de generación de vapor.

Durante los últimos 30 años el diseño de las calderas pirotubulares ha experimentado mejoras en todas direcciones, necesitando una calidad superior del agua de alimentación. Estas calderas operan a elevadas tasas de transferencia de calor y tienen capacidades de evaporación en el rango de 10000 kg/h. Los dos problemas más comunes encontrados bajo tales condiciones son las deposiciones y la corrosión [3]. El acondicionamiento químico del agua de alimentación a los generadores de vapor tiene como objetivo evitar la corrosión de los tubos internos y prevenir la formación de depósitos en las superficies de transferencia de calor. En el caso de la dureza total (DT) del agua, este es un parámetro clave que necesita ser controlado bajo ciertos límites para evitar una reducción de la eficiencia energética de la caldera, sobreconsumo de combustible, roturas de los tubos y deficiencias importantes en los procesos de transferencia de calor. Según [10], la DT del agua de alimentación de calderas pirotubulares operando a una presión de $0-20 \mathrm{kgf} / \mathrm{cm}^{2}$ debe ser $\leq 1,0 \mathrm{ppm}$; en [11] se indica que la DT del agua deberá ser de hasta 2,0 ppm para calderas pirotubulares que operan hasta $30 \mathrm{kgf} / \mathrm{cm}^{2}$; mientras que por último en [12] se especifica que la DT para calderas pirotubulares operando a presiones $\leq 40 \mathrm{kgf} / \mathrm{cm}^{2}$ deberá ser $<1$ ppm.

En el presente trabajo se evaluó el sistema de generación y distribución de vapor Combinado Cárnico "César Escalante Dellundé" de la provincia de Camagüey, Cuba. Se determinó la eficiencia energética de la caldera pirotubular instalada, las pérdidas de calor al ambiente de las tuberías sin aislamiento, y la dureza total de agua de alimentación a la caldera. Se proponen dos alternativas de mejoras para incrementar la eficiencia energética del generador de vapor y reducir el consumo de combustible, las cuales consisten en: 1) aislamiento de las tuberías por donde circula vapor de agua y 2) precalentamiento del agua de alimentación al generador de vapor hasta $70{ }^{\circ} \mathrm{C}$ mediante un sistema de control automático. Se determinó el impacto económico que presentan ambas alternativas con respecto al ahorro de combustible obtenido, asi como también el período de recuperación de la inversión necesario para amortizar el costo total de inversión de ambas propuestas.

\section{MATERIALES Y MÉTODOS}

A. Descripción del proceso de generación, distribución y consumo de vapor de agua en la empresa cárnica

Primeramente, el combustible se recibe en un tanque de almacenamiento con una capacidad de 39862 litros para entonces, mediante la bomba de combustible de primer impulso, se envía hacia el tanque diario de la caldera (tanque de trabajo) donde se precalienta utilizando vapor de agua como medio de calentamiento. Después se bombea el combustible hacia otro sistema de precalentamiento mediante la bomba de segundo 
impulso, hasta lograr aproximadamente $90{ }^{\circ} \mathrm{C}$. Con esta temperatura se alimenta hacia el quemador de la caldera. En este sistema de precalentamiento primero se calienta el combustible a través de una resistencia eléctrica, y una vez que esté caliente y la caldera este generando vapor es que se realiza el 1er precalentamiento.

El agua llega desde la extracción de la presa para la cisterna y mediante un sistema de bombeo se lleva hasta el tanque elevado, donde luego, debido a la presión de la gravedad, se distribuye hacia toda la industria. En el caso del consumo de agua en la caldera, el agua pasa por los suavizadores y luego se envía hacia el tanque de almacenamiento de la caldera, para poder bombearla hacia su interior. El vapor saturado producido en la caldera, a una presión de $8 \mathrm{kgf} / \mathrm{cm}^{2}$, es enviado hacia un distribuidor de vapor (Manifold), mediante el cual se alimentan todas las áreas consumidoras de vapor de la fábrica, las cuales son:

- Área del matadero de vacas

- Área del matadero de cerdo

- Área de embutidos

- Área de croquetas

- Área de hornos y tachos

En la Fig. 1 se muestra el sistema de generación y distribución de vapor en el área de matadero de cerdos del Combinado Cárnico.

\section{B. Características del generador de vapor}

En el Combinado Cárnico existe actualmente en funcionamiento un generador de vapor modelo $\mathrm{CMS} / \mathrm{C}-5000$,

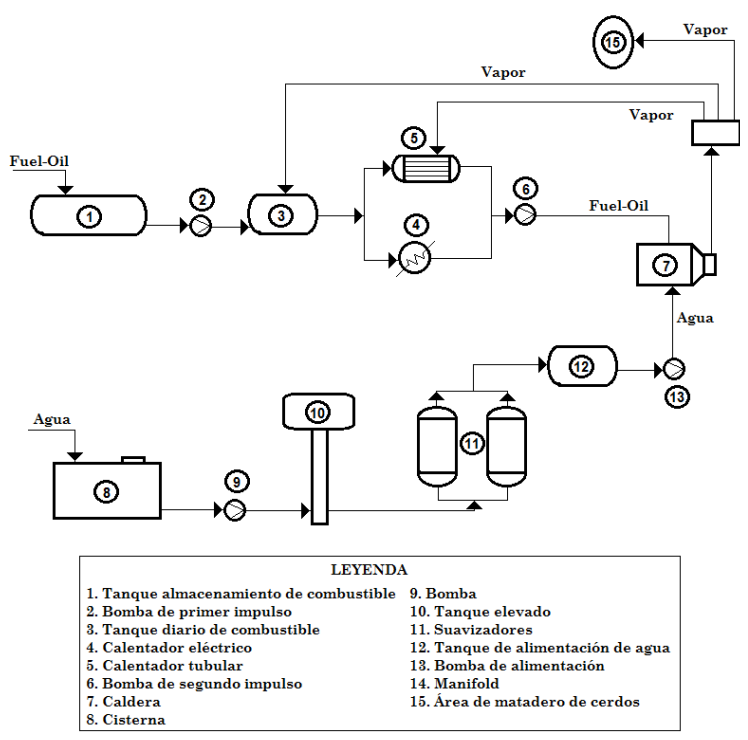

Fig. 1. Diagrama de flujo del sistema de generación de vapor del Combinado Cárnico.

En la instalación se trabaja con agua dura, que no son más que aquellas que llevan una gran cantidad de sales calcáreas en solución y que, al vaporizar precipitan formando incrustaciones fuertemente adheridas a las planchas y tubos de la caldera, dando como resultado un mayor consumo de combustible al del tipo pirotubular, fabricado en el año 1979 y puesto en marcha en abril del 2017. Tiene una capacidad de producción de vapor de $5000 \mathrm{~kg} / \mathrm{h}$, una presión de salida del vapor 8 $\mathrm{kgf} / \mathrm{cm}^{2}$, por lo que se considera una caldera de baja presión [3], además de un índice nominal de generación de vapor de $15 \mathrm{~kg}$ de vapor/kg de combustible, consumiendo fundamentalmente Fuel-Oil como combustible. Esta caldera posee una superficie de calefacción de $143,7 \mathrm{~m}^{2}$, una altura de 3,540 m y un largo de 7,050 m.

El quemador trabaja a una presión normal de $3 \mathrm{kgf} / \mathrm{cm}^{2}$, la temperatura de alimentación del combustible precalentado es de $100{ }^{\circ} \mathrm{C}$, y el aire es inyectado bajo tiro forzado. La temperatura de salida de los gases por la chimenea es de $180-200{ }^{\circ} \mathrm{C}$, siendo la temperatura de trabajo del vapor de agua de $169,6{ }^{\circ} \mathrm{C}$.

Antes del encendido de la caldera los operadores deben verificar la existencia de la cantidad necesaria de agua tratada $\left(6 \mathrm{~m}^{3}\right)$ y de combustible $(3574 \mathrm{~L})$, además del correcto funcionamiento de todo el equipamiento del área.

Según datos facilitados por personal de dirección de la instalación, el generador de vapor trabaja un promedio de 283 días al año, y 12 horas al día.

\section{Determinación de la dureza total del agua de alimentación a la caldera}

El agua de alimentación es uno de los factores más importantes que influyen en el buen funcionamiento de la caldera, pues de él depende el rendimiento, la vida del mismo y gran parte de las averías que se puedan presentar.

dificultar la transmisión de calor. De acuerdo con criterios e informaciones suministradas por especialistas de la empresa ALASTOR, se ha comprobado experimentalmente que un espesor de 6 $\mathrm{mm}$ de incrustaciones en los tubos de una caldera exige un $50 \%$ de más combustible para una misma producción de vapor.

Para determinar la dureza total (DT) del agua suave antes de ser alimentada al generador de vapor, se empleó la metodología vigente existente para ello [13]. Se tomaron 2 muestras de $50 \mathrm{~mL}$ del agua objetivo y se le adicionaron $5 \mathrm{~mL}$ de una solución tampón $(\mathrm{pH}=10)$, así como también una pizca del indicador Negro Eriocromo T (NET), obteniéndose una solución de color rojo vino. A continuación, se valoró la solución obtenida con una solución de ácido etilén-diamintetracético (EDTA) de concentración 0,01 mol/L previamente colocada en una bureta, hasta que se obtuvo una coloración azul. La DT se determinó de acuerdo con la siguiente ecuación:

$$
c_{\left(\mathrm{Ca}^{2+}+\mathrm{Mg}^{2+}\right)_{N}}=\frac{c_{(E D T A)} \cdot V_{(E D T A)}}{V_{(H 2 O)}}
$$

Por su parte, la dureza cálcica se determinó mediante la siguiente metodología [14]. Se pipetearon dos alícuotas de 50,0 mL de la muestra de agua y se adicionaron $2 \mathrm{~mL}$ de hidróxido de sodio $(\mathrm{NaOH})$ a cada 
una, además de una pizca del indicador Murexida, obteniéndose una solución de coloración rojo fresa. A continuación, se valoró la solución obtenida con una solución de EDTA de concentración 0,01 mol/L previamente colocada en una bureta, hasta obtener una coloración violeta. La concentración de iones calcio se determinó mediante (2):

$$
c_{\left(C a^{2+}\right)_{N}}=\frac{c_{(E D T A)} \cdot V_{(E D T A)}}{V_{(H 2 O)}}
$$

Por último, la dureza magnésica se calculó según (3):

$$
c_{\left(\mathrm{Mg}^{2+}\right)_{N}}=c_{\left(\mathrm{Ca}^{2+}+\mathrm{Mg}^{2+}\right)_{N}}-c_{\left(\mathrm{Ca}^{2+}\right)_{N}}
$$

Luego, para determinar la dureza cálcica, magnésica y total en unidades de ppm se utilizaron (4) (5) y (6), respectivamente:

$$
\begin{aligned}
& c_{\left(\mathrm{Ca}^{2+}\right)_{\mathrm{ppm}}}=c_{\left(\mathrm{Ca}^{2+}\right)_{N}} \cdot M_{(\mathrm{Ca})} \cdot 1000 \\
& c_{\left(\mathrm{Mg}^{2+}\right)_{\mathrm{ppm}}}=c_{\left(\mathrm{Mg}^{2+}\right)_{N}} \cdot M_{(\mathrm{Mg})} \cdot 1000 \\
& c_{\left(\mathrm{Ca}^{2+}+\mathrm{Mg}^{2+}\right)_{\mathrm{ppm}}}=c_{\left(\mathrm{Ca}^{2+}\right)_{\mathrm{ppm}}}+c_{\left(\mathrm{Mg}^{2+}\right)_{\mathrm{ppm}}}
\end{aligned}
$$

El valor promedio obtenido de la DT para el agua de alimentación a la caldera será comparado con el límite máximo establecido por la norma británica BS: 2486 [11], con el fin de evaluar el funcionamiento del sistema de ablandamiento de agua de la instalación.

D. Determinación de las pérdidas de calor al ambiente en las tuberías de vapor de agua y cálculo del espesor óptimo del aislante.

Para determinar las pérdidas de calor al ambiente en las tuberías por donde fluye vapor de agua primeramente se realizó un levantamiento hidráulico bien detallado del sistema de redes, el cual fue dividido en tramos para conocer sus características (orientación horizontal o vertical, longitud, diámetro interior y exterior) y luego proceder a los cálculos necesarios. En la Tabla I se muestra el levantamiento hidráulico efectuado para los diferentes tramos de tubería por donde circula el vapor de agua. 
TABLA I.

LEVANTAMIENTO HIDRÁULICO DE LAS REDES DE TUBERÍAS DE DISTRIBUCIÓN DE VAPOR DE AGUA EN CONDICIONES ACTUALES.

\begin{tabular}{|c|c|c|c|c|c|c|}
\hline No. & Tramo de tubería & $\begin{array}{l}\text { Longitud total } \\
(\mathrm{m})\end{array}$ & $\begin{array}{l}\text { Horizontal } \\
\quad(\mathrm{m})\end{array}$ & $\begin{array}{l}\text { Vertical } \\
(\mathrm{m})\end{array}$ & $\begin{array}{l}\text { Diámetro Interior } \\
(\mathrm{mm})\end{array}$ & $\begin{array}{l}\text { Diámetro exterior } \\
(\mathrm{mm})\end{array}$ \\
\hline 1 & $\begin{array}{l}\text { Manifold de la caldera - } \\
\text { Calentador de combustible }\end{array}$ & 13,5 & 7,5 & 6 & 62,71 & 73,15 \\
\hline 2 & $\begin{array}{l}\text { Manifold de la caldera- tanque } \\
\text { de agua de alimentar la caldera }\end{array}$ & 23,25 & 10,1 & 13,15 & 15,80 & 21,34 \\
\hline 3 & $\begin{array}{l}\text { Manifold de la caldera- } \\
\text { Matadero de cerdos }\end{array}$ & 124,20 & 110,20 & 7 & 52,50 & 60,45 \\
\hline $3 a$ & $\begin{array}{l}\text { Manifold de la caldera- } \\
\text { Matadero de cerdos }\end{array}$ & 257,25 & 245,25 & 12 & 26,64 & 33,53 \\
\hline
\end{tabular}

La determinación de las pérdidas de calor al ambiente en las tuberías por donde circula vapor, así como también el cálculo del espesor óptimo del aislante, se efectuó utilizando una hoja de cálculo Excel [15], la cual está basada en la metodología publicada en [16]. Este programa requiere los siguientes datos iniciales:

- Diámetro exterior de la tubería [m]

- Diámetro interior de la tubería [m]

- Longitud total de la tubería (para el caso de tuberías horizontales) [m]

- Temperatura del aire exterior $\left[{ }^{\circ} \mathrm{C}\right]$

- Temperatura del fluido interior de la tubería $\left[{ }^{\circ} \mathrm{C}\right]$

- Altura (para el caso de tuberías verticales) $\left[{ }^{\circ} \mathrm{C}\right]$

- Emisividad total normal de la tubería

Se propuso aislar las tuberías con el aislante Lana Mineral, el cual se recubrirá exteriormente con chapa de aluminio.

E. Determinación de la eficiencia energética del generador de vapor

La eficiencia energética del generador de vapor fue determinada mediante el método indirecto utilizando una hoja de cálculo Excel [17], la cual se basa en la metodología de cálculo planteada por [18]. Este programa exige que se dispongan los siguientes datos iniciales:

- Producción de vapor $[\mathrm{t} / \mathrm{h}]$.

- $\%$ de $\mathrm{CO}_{2}$ en los gases de combustión.

- $\%$ de $\mathrm{O}_{2}$ en los gases de combustión.

- $\%$ de $\mathrm{CO}$ en los gases de combustión.

- Índice de Bacharach.

- Presión del vapor $\left[\mathrm{kgf} / \mathrm{cm}^{2}\right]$.

- Temperatura de salida de los gases $\left[{ }^{\circ} \mathrm{C}\right]$.

- Temperatura exterior de la caldera $\left[{ }^{\circ} \mathrm{C}\right]$.

- Temperatura del agua de alimentación $\left[{ }^{\circ} \mathrm{C}\right]$.

- Temperatura del vapor $\left[{ }^{\circ} \mathrm{C}\right]$.

- Temperatura del aire ambiente $\left[{ }^{\circ} \mathrm{C}\right]$.

- Humedad relativa del aire [\%].

- Parámetros físicos-químicos del combustible utilizado (Fuel-Oil).

- Características del generador de vapor: (altura, diámetro, largo, ancho, material exterior y su coeficiente de emisividad).
F. Propuesta de alternativas de mejoras para incrementar la eficiencia energética en el generador de vapor

$\mathrm{Se}$ propondrán dos alternativas de mejoras para incrementar la eficiencia energética del generador de vapor, las cuales se relacionan a continuación.

Propuesta 1: Aislamiento total de las tuberías de vapor

Como bien se explicó con anterioridad, se propone aislar todas las redes de tuberías por donde circula vapor de agua con el aislante Lana Mineral y recubrimiento de aluminio, lo cual tendrá un gran impacto técnico-económico y medioambiental en el proceso de generación de vapor de la fábrica. Esto se debe a la reducción de pérdidas de calor al ambiente, lo cual trae consigo un ahorro en el consumo de combustible, y por ende, una reducción del gasto económico, además de una disminución de la emisión de gases contaminantes a la atmósfera. Es válido recordar que la temperatura no permanece constante por toda la tubería, ya que esta depende del lugar donde se encuentre la instalación, sea en el interior o el exterior de las edificaciones.

Propuesta 2: Precalentamiento del agua de alimentación al generador de vapor

En el área de generación de vapor existe un tanque horizontal con una capacidad de $15 \mathrm{~m}^{3}$, el cual se emplea para el almacenamiento del agua tratada que se consume en el generador (Fig. 2). Actualmente existe una tubería de hierro de 1/2 pulg de diámetro nominal, la cual va desde el Manifold hasta el tanque del agua de alimentación de agua, y es la encargada de suministrar el vapor de dicho tanque para precalentar el agua. No existe un control automático para posibilitar que se alcance una temperatura estable de aproximadamente entre $60-70{ }^{\circ} \mathrm{C}$ para el agua contenida en este tanque, por lo que se propone la instalación de un controlador proporcional derivativo y una válvula de retención tipo charnela de rosca hembra, para que se posibilite un control automatizado del flujo de vapor desde el Manifold hasta el tanque, lo cual facilite, a su vez, que se alcance y mantenga la temperatura del agua en el interior del tanque en el rango de temperatura deseado $\left(60-70^{\circ} \mathrm{C}\right)$. 


$$
M_{\mathrm{CO}_{2}}^{a}=I_{g} \cdot x_{\mathrm{CO}_{2}} \cdot F_{c}^{a} \cdot t_{a \tilde{n} o} \cdot t_{d i ́ a}
$$

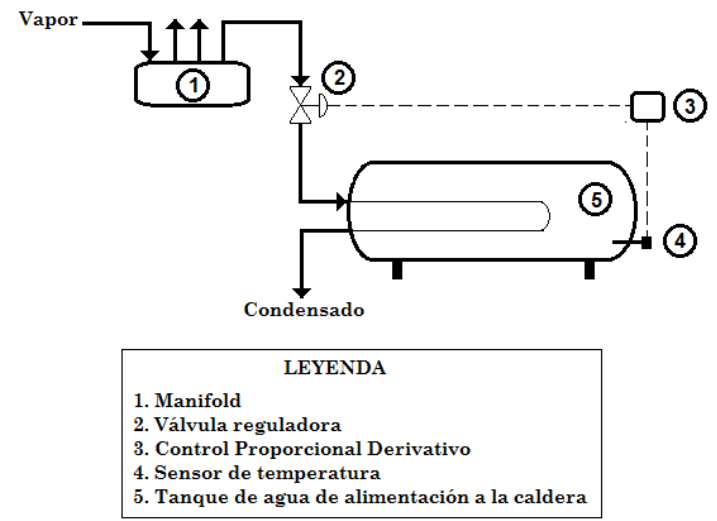

Fig. 2. Diagrama de la propuesta del sistema de precalentamiento del agua de alimentación al generador de vapor.

\section{G. Comportamiento medioambiental}

Para efectuar los cálculos medioambientales se emplearon los valores obtenidos en la hoja de cálculo Excel utilizada para determinar la eficiencia energética del generador de vapor [16], a partir de la cual se obtuvo la cantidad de gases producidos por cada kilogramo de combustible consumido. De esta manera se determinó el caudal másico anual de cada uno de los componentes presentes en los gases de combustión, tanto para las condiciones actuales como considerando las mejoras. Por tanto, la cantidad de gases emitidos por año por componente en las condiciones actuales se determinó mediante las siguientes ecuaciones:

Cantidad total de gases emitidos por año:

$$
M_{g}^{a}=I_{g} \cdot F_{c}^{a} \cdot t_{\text {año }} \cdot t_{\text {día }}
$$

Dónde:

$M_{g}^{a}$ - Flujo másico total de gases de combustión emitidos por año en las condiciones actuales [kg/año]. $I_{g}$ - Cantidad de gases de combustión producidos por cada kilogramo de combustible consumido en el generador de vapor $\left[\mathrm{kg}_{\text {gases }} / \mathrm{kg}_{\text {combustible }}\right]$. Este dato lo suministra la hoja de cálculo Excel utilizada para determinar la eficiencia energética del generador de vapor [17].

$F_{c}^{a}$

- Caudal másico de combustible que se alimenta al generador de vapor bajo las condiciones actuales $[\mathrm{kg} / \mathrm{h}]$.
- $t_{a \tilde{n} o}$ - Días que trabaja el generador de vapor por año [días/año]

- $\quad t_{\text {día }}$ - Horas que trabaja el generador de vapor por día [h/día]

Cantidad de dióxido de carbono $\left(\mathrm{CO}_{2}\right)$ emitido por año:

$M_{N_{2}}^{a}=I_{g} \cdot x_{N_{2}} \cdot F_{c}^{a} \cdot t_{\text {año }} \cdot t_{\text {día }}$

Dónde:

- $\quad \mathrm{M}_{\mathrm{CO}_{2}}^{a}$ - Flujo másico de $\mathrm{CO}_{2}$ emitido por año para las condiciones actuales $\left[\mathrm{kgCO}_{2} / \mathrm{año}\right]$

- $x_{\mathrm{CO}_{2}}$ - Fracción másica de $\mathrm{CO}_{2}$ en los gases de combustión.

Cantidad de nitrógeno $\left(\mathrm{N}_{2}\right)$ emitido por año:

Dónde:

- $\quad M_{N_{2}}^{a}$ - Flujo másico de $\mathrm{N}_{2}$ emitido por año para las condiciones actuales $\left[\mathrm{kgN}_{2} / \mathrm{año}\right]$.

- $x_{N_{2}}$ - Fracción másica de $\mathrm{N}_{2}$ en los gases de combustión.

Cantidad de oxígeno $\left(\mathrm{O}_{2}\right)$ emitido por año:

$$
M_{O_{2}}^{a}=I_{g} \cdot x_{O_{2}} \cdot F_{c}^{a} \cdot t_{\text {año }} \cdot t_{\text {día }}
$$

Dónde:

- $\quad M_{O_{2}}^{a}$ - Flujo másico de $\mathrm{O}_{2}$ emitido por año para las condiciones actuales $\left[\mathrm{kgO}_{2} / \mathrm{año}\right]$.

- $x_{O_{2}}$ - Fracción másica de $\mathrm{O}_{2}$ en los gases de combustión.

Cantidad de óxido de azufre $\left(\mathrm{SO}_{2}\right)$ emitido por año:

$$
M_{S O_{2}}^{a}=I_{g} \cdot x_{S O_{2}} \cdot F_{c}^{a} \cdot t_{\text {año }} \cdot t_{\text {día }}
$$

Dónde:

- $\quad \mathrm{M}_{\mathrm{SO}_{2}}^{a}$ - Flujo másico de $\mathrm{SO}_{2}$ emitido por año para las condiciones actuales $\left[\mathrm{kgSO}_{2} / \mathrm{año}\right]$.

- $x_{\mathrm{SO}_{2}}$ - Fracción másica de $\mathrm{SO}_{2}$ en los gases de combustión.

Luego se halló la cantidad de gases emitidos al ambiente, tanto total como por componente, considerando las mejoras:

$$
M_{g}^{m}=I_{g} \cdot F_{c}^{m} \cdot t_{\text {año }} \cdot t_{\text {día }}
$$

Dónde:

- $\quad M_{g}^{m}$ - Flujo másico total de gases de combustión emitidos por año considerando mejoras [kg/año].

- $\quad F_{c}^{m}$ - Caudal másico de combustible que se alimenta al generador de vapor considerando mejoras $[\mathrm{kg} / \mathrm{h}]$.

Cantidad de $\mathrm{CO}_{2}$ emitido por año:

$$
M_{\mathrm{CO}_{2}}^{m}=I_{g} \cdot x_{\mathrm{CO}_{2}} \cdot F_{c}^{m} \cdot t_{\text {año }} \cdot t_{\text {día }}
$$

Dónde: 
- $\quad M_{\mathrm{CO}_{2}}^{m}-$ Flujo másico de $\mathrm{CO}_{2}$ emitido por año considerando mejoras $\left[\mathrm{kgCO}_{2} / \mathrm{año}\right]$.

Cantidad de $\mathrm{N}_{2}$ emitido por año:

$$
M_{N_{2}}^{m}=I_{g} \cdot x_{N_{2}} \cdot F_{c}^{m} \cdot t_{\text {año }} \cdot t_{\text {día }}
$$

Dónde:

$$
\begin{aligned}
& M_{N_{2}-}^{m} \text { Flujo másico de } \mathrm{N}_{2} \text { emitido por año } \\
& \text { considerando mejoras }\left[\mathrm{kgN}_{2} / \mathrm{año}\right] \text {. }
\end{aligned}
$$

Cantidad de $\mathrm{O}_{2}$ emitido por año:

$$
M_{O_{2}}^{m}=I_{g} \cdot x_{O_{2}} \cdot F_{c}^{m} \cdot t_{\text {año }} \cdot t_{\text {día }}
$$

Dónde:

$$
\begin{aligned}
& M_{O_{2}}^{m}-\text { Flujo másico de } \mathrm{O}_{2} \text { emitido por año } \\
& \text { considerando mejoras }\left[\mathrm{kgO}_{2} / \mathrm{año}\right] \text {. }
\end{aligned}
$$

Cantidad de $\mathrm{SO}_{2}$ emitido por año:

$$
M_{S O_{2}}^{m}=I_{g} \cdot x_{S_{2}} \cdot F_{c}^{m} \cdot t_{a \tilde{n} o} \cdot t_{\text {día }}
$$

Dónde:

$$
\begin{aligned}
& M_{\mathrm{SO}_{2}}^{m} \text { - Flujo másico de } \mathrm{SO}_{2} \text { emitido por año } \\
& \text { considerando mejoras }\left[\mathrm{kgSO}_{2} / \mathrm{año}\right] \text {. }
\end{aligned}
$$

Por último, la cantidad de gases de combustión dejados de emitir a la atmósfera producto a aplicación de las mejoras propuestas se determinó mediante las ecuaciones que se muestran a continuación:

Ahorro total de gases de combustión que no se emiten a la atmósfera producto a la implementación de las mejoras:

$$
M_{g}^{a h}=M_{g}^{a}-M_{g}^{m}
$$

Ahorro por componente:

- Dióxido de carbono:

$$
M_{\mathrm{CO}_{2}}^{a h}=M_{\mathrm{CO}_{2}}^{a}-M_{\mathrm{CO}_{2}}^{m}
$$

- Nitrógeno:

$$
\begin{aligned}
& M_{N_{2}}^{a h}=M_{N_{2}}^{a}-M_{N_{2}}^{m} \\
& \text { Oxígeno: } \\
& M_{O_{2}}^{a h}=M_{O_{2}}^{a}-M_{O_{2}}^{m}
\end{aligned}
$$

- Dióxido de azufre:

$$
M_{S O_{2}}^{a h}=M_{S O_{2}}^{a}-M_{S O_{2}}^{m}
$$

H. Cálculo de la dispersión de los gases contaminantes

Para determinar la dispersión de los gases contaminantes producto de la combustión se empleó el software Contamin [19], mediante el cual se pueden estimar la concentración máxima de contaminantes emitidos a la atmósfera debido al proceso de combustión, y la distancia máxima a la que los gases alcanzan su mayor concentración. Este software requiere de los siguientes datos iniciales:

- $\quad$ Altura de la chimenea $=9 \mathrm{~m}$.

- Diámetro de la chimenea $=0,075 \mathrm{~m}$.
- Flujo volumétrico de la mezcla de gases de combustión $\left[\mathrm{m}^{3} / \mathrm{s}\right]$.

- Flujo másico de la sustancia contaminante [g/s].

- Temperatura de los gases de combustión = 453 K.

- $\quad$ Temperatura del aire ambiente $=303 \mathrm{~K}$.

De todos los datos iniciales relacionados con anterioridad, solamente no se disponen el flujo volumétrico de la mezcla de gases de combustión (en $\mathrm{m}^{3} / \mathrm{s}$ ), y el flujo másico de la sustancia contaminante (en $\mathrm{g} / \mathrm{s}$ ), los cuales deben ser determinados tanto para las condiciones actuales como considerando la aplicación de las mejoras propuestas.

El flujo volumétrico de la mezcla de los gases de combustión, tanto para las condiciones actuales como para las mejoras propuestas, se determinó mediante la siguiente metodología.

Paso 1. Determinación de la densidad de cada uno de los compuestos presentes en los gases de combustión a la temperatura de salida de la mezcla de gases en la chimenea

Se empleará la ecuación de Clapeyron [20]:

$$
\rho_{i}=\frac{M_{i}}{22,4} \cdot \frac{273 \cdot P_{i}}{T_{g} \cdot P_{0}}
$$

Dónde:

- $\quad \mathrm{M}_{\mathrm{i}}$ - Masa molar del componente $\mathrm{i}[\mathrm{kg} / \mathrm{kmol}]$

- $\mathrm{T}_{\mathrm{g}}$ - Temperatura de los gases de combustión $=453,15$ $\mathrm{K}$

- $\mathrm{P}_{\mathrm{i}}-$ Presión del componente $\mathrm{i}$ en los gases de combustión $=1$ atm.

- $\quad \mathrm{P}_{0}-$ Presión de referencia $=1$ atm

De esta manera, la densidad del $\mathrm{CO}_{2}, \mathrm{~N}_{2}, \mathrm{O}_{2}$ y $\mathrm{SO}_{2}$ en los gases de combustión se determinó mediante $(23)(24)(25)$ y (26), respectivamente.

$\rho_{\mathrm{CO}_{2}}=\frac{M_{\mathrm{CO}_{2}}}{22,4} \cdot \frac{273 \cdot P_{\mathrm{CO}_{2}}}{T_{g} \cdot P_{0}}$

$\rho_{N_{2}}=\frac{M_{N_{2}}}{22,4} \cdot \frac{273 \cdot P_{N_{2}}}{T_{g} \cdot P_{0}}$

$\rho_{O_{2}}=\frac{M_{O_{2}}}{22,4} \cdot \frac{273 \cdot P_{O_{2}}}{T_{g} \cdot P_{0}}$

$\rho_{\mathrm{SO}_{2}}=\frac{M_{\mathrm{SO}_{2}}}{22,4} \cdot \frac{273 \cdot P_{\mathrm{SO}_{2}}}{T_{g} \cdot P_{0}}$

Paso 2. Cálculo de la densidad de la mezcla de gases de combustión a la temperatura de salida en la chimenea.

Se empleará la siguiente ecuación [20]:

$\rho_{g}=\left(\rho_{\mathrm{CO}_{2}} \cdot y_{\mathrm{CO}_{2}}\right)+\left(\rho_{\mathrm{N}_{2}} \cdot y_{N_{2}}\right)$

$+\left(\rho_{\mathrm{O}_{2}} \cdot y_{\mathrm{O}_{2}}\right)+\left(\rho_{\mathrm{SO}_{2}} \cdot y_{\mathrm{SO}_{2}}\right)$ 
Dónde:

УCO2; УN2; yo2 y ysO2 - Fracción volumen del $\mathrm{CO}_{2}, \mathrm{~N}_{2}, \mathrm{O}_{2}$ y $\mathrm{SO}_{2}$ en los gases de combustión, respectivamente. Estos datos los proporciona la hoja de cálculo Excel utilizada para determinar la eficiencia energética de la caldera [16].

Paso 3. Determinación del flujo volumétrico de la mezcla de gases de combustión ( $\mathrm{Vg}$ ):

Para las condiciones actuales:

$$
V_{g}^{a}=\frac{I_{g} \cdot F_{c}^{a}}{3600 \cdot \rho_{g}}
$$

Considerando mejoras:

$$
V_{g}^{m}=\frac{I_{g} \cdot F_{c}^{m}}{3600 \cdot \rho_{g}}
$$

Por su parte, para calcular el flujo másico de cada componente presente en los gases de combustión, tanto para las condiciones actuales como para la implementación de las mejoras, se utilizarán las siguientes ecuaciones:

Condiciones actuales:

- Dióxido de carbono:

$$
m_{\mathrm{CO}_{2}}^{a}=\frac{I_{g} \cdot x_{\mathrm{CO}_{2}} \cdot F_{c}^{a} \cdot 1000}{3600}
$$

- Nitrógeno:

$m_{N_{2}}^{a}=\frac{I_{g} \cdot x_{N_{2}} \cdot F_{c}^{a} \cdot 1000}{3600}$

- Oxígeno:

$m_{O_{2}}^{a}=\frac{I_{g} \cdot x_{O_{2}} \cdot F_{c}^{a} \cdot 1000}{3600}$

- Dióxido de azufre:

$$
m_{\mathrm{SO}_{2}}^{a}=\frac{I_{g} \cdot x_{\mathrm{SO}_{2}} \cdot F_{c}^{a} \cdot 1000}{3600}
$$

Considerando las mejoras propuestas:

- Dióxido de carbono:

$$
m_{\mathrm{CO}_{2}}^{m}=\frac{I_{g} \cdot x_{\mathrm{CO}_{2}} \cdot F_{c}^{m} \cdot 1000}{3600}
$$

- Nitrógeno:

$m_{N_{2}}^{m}=\frac{I_{g} \cdot x_{N_{2}} \cdot F_{c}^{m} \cdot 1000}{3600}$

- Oxígeno:

$m_{O_{2}}^{m}=\frac{I_{g} \cdot x_{O_{2}} \cdot F_{c}^{m} \cdot 1000}{3600}$

\section{Cálculos económicos}

A continuación se muestran las metodologías utilizadas para determinar los siguientes parámetros económicos:

1. Costo de inversión del aislamiento de las tuberías por donde circula vapor.

2. Costo de inversión del sistema de precalentamiento del agua al generador de vapor.

3. Costo total de inversión de ambas propuestas de mejoras.

4. Ahorro económico que se obtiene debido a combustible que no se consume en el generador de vapor producto a la implementación del sistema de precalentamiento del agua de alimentación.

5. Ahorro económico que se obtiene debido a combustible que no se consume en el generador de vapor producto al adecuado aislamiento de las tuberías por donde circula vapor de agua.

6. Período de recuperación de la inversión.

1) Costo de inversión relacionado con el aislamiento de las tuberías por donde circula vapor de agua

Según la empresa suministradora del material aislante (Alastor), la Lana Mineral con recubrimiento de aluminio presenta el precio unitario que se muestran en la Tabla II.

TABLA II.

PRECIO UNITARIO DEL MATERIAL AISLANTE LANA MINERAL JUNTO CON RECUBRIMIENTO DE ALUMINIO SEGÚN LA EMPRESA SUMINISTRADORA ALASTOR.

\begin{tabular}{ccc}
\hline \hline $\begin{array}{l}\text { Diámetro nominal del tubo } \\
\text { (pulg) }\end{array}$ & $\begin{array}{c}\text { Espesor } \\
(\mathrm{mm})\end{array}$ & $\begin{array}{c}\text { Precio } \\
(\mathrm{PC} \$ / \mathrm{m})\end{array}$ \\
\hline $1 / 2$ & 25 & 3,58 \\
1 & 25 & 6,80 \\
2 & 40 & 7,27 \\
$21 / 2$ & 40 & 20,06 \\
\hline \hline
\end{tabular}

El costo de aislamiento de las tuberías por donde circula vapor se determina según la siguiente ecuación:

$$
\begin{aligned}
& C_{\text {aisl }}=\left[\left(L_{t u b}^{0,5} \cdot P_{a i s l}^{0,5}\right)+\left(L_{t u b}^{1} \cdot P_{a i s l}^{1}\right)\right. \\
& \left.+\left(L_{t u b}^{2} \cdot P_{a i s l}^{2}\right)+\left(L_{t u b}^{2,5} \cdot P_{a i s l}^{2.5}\right)\right]
\end{aligned}
$$

Dónde:

- $\quad L_{t u b}^{0,5}$ - Longitud de las tuberías con un diámetro de $1 / 2$ pulg [m].

- $\quad P_{\text {aisl }}^{0,5}$ Precio del material aislante para una tubería de $1 / 2$ pulg $[\mathrm{PC} \$ / \mathrm{m}]$.

- $\quad L_{t u b}^{1}$ - Longitud de las tuberías con un diámetro de 1 pulg $[\mathrm{m}]$.

- $\quad P_{a i s l}^{1}$ - Precio del material aislante para una tubería de 1 pulg $[\mathrm{PC} \$ / \mathrm{m}]$.

- Dióxido de azufre: 
- $\quad L_{t u b}^{2}$ - Longitud de las tuberías con un diámetro de 2 pulg $[\mathrm{m}]$.

- $\quad P_{\text {aisl }}^{2}$ - Precio del material aislante para una tubería de 2 pulg $[\mathrm{PC} \$ / \mathrm{m}]$.

- $\quad L_{t u b}^{2,5}$ - Longitud de las tuberías con un diámetro de $2 \frac{1 / 2}{2}$ pulg $[\mathrm{m}]$.

- $\quad P_{\text {aisl }}^{2.5}$ Precio del material aislante para una tubería de
$21 / 2$ pulg [PC $\$ / \mathrm{m}]$.

$\mathrm{Al}$ costo de adquisición de los materiales aislantes $\left(\mathrm{C}_{\text {aisl }}\right)$ se le debe agregar el costo de su instalación $\left(\mathrm{C}_{\text {inst }}\right)$, el cual será de un $2 \%$ del costo total de adquisición según [21] para una operación con este grado de complejidad. De esta manera, el costo de inversión total debido al aislamiento de las tuberías por donde fluye vapor de agua con Lana Mineral será de:

$$
\begin{aligned}
& C_{\text {inv }}^{\text {aisl }}=C_{\text {aisl }}+C_{\text {inst }} \\
& C_{\text {inv }}^{\text {aisl }}=C_{\text {aisl }}+C_{\text {aisl }} \cdot 0,02
\end{aligned}
$$

2) Costo de inversión del sistema de regulación del precalentamiento del agua de alimentación al generador de vapor

Para la instalación del sistema de precalentamiento del agua de alimentación del generador de vapor solo se requiere de la adquisición y montaje del Controlador Proporcional Derivativo, que abarca el sensor de temperatura, el controlador en sí y la válvula de regulación automática del vapor, lo cual alcanza un costo total de \$ 3750 Pesos Cubanos (PC) según consultas realizadas al personal de la empresa especializada Alastor. Es decir:

$$
C_{i n v}^{\text {precal }}=\operatorname{PC} \$ 3750
$$

\section{3) Costo de inversión total}

El costo total de inversión de ambas propuestas de mejoras será determinado mediante la siguiente ecuación:

$$
C_{i n v}^{\text {total }}=C_{i n v}^{\text {aisl }}+C_{i n v}^{\text {precal }}
$$

4) Ahorro económico anual obtenido debido a la

$$
m_{\mathrm{SO}_{2}}^{m}=\frac{I_{g} \cdot x_{\mathrm{SO}_{2}} \cdot F_{c}^{m} \cdot 1000}{3600}
$$

implementación del sistema de precalentamiento del agua de alimentación al generador de vapor

El ahorro económico anual obtenido debido a la implementación del sistema de precalentamiento de agua de alimentación al generador de vapor se determinará mediante la siguiente ecuación:

$$
A_{\text {precal }}=\frac{A_{c o m b} \cdot t_{\text {día }} \cdot t_{a \tilde{n o}} \cdot C_{c o m b}}{\rho_{c o m b}}
$$

Dónde:

- $A_{\text {precal }}$ - Ahorro económico anual obtenido debido a la implementación del sistema de precalentamiento del agua de alimentación al generador de vapor [\$/año].

- $A_{\text {comb }}$ - Ahorro de combustible obtenido producto al precalentamiento del agua de alimentación al generador de vapor hasta $70{ }^{\circ} \mathrm{C}[\mathrm{kg} / \mathrm{h}]$. Se obtiene mediante la hoja de cálculo Excel utilizada para determinar la eficiencia energética del generador de vapor [17].

- $t_{\text {día }}$ - Horas que trabaja la caldera por día $=12 \mathrm{~h} /$ día.

- $\quad t_{a \tilde{n} o}$ - Días que opera la caldera por año = 283 día/año.

- $C_{\text {comb }}$ - Costo del combustible $=$ PC $\$ 0,39 / \mathrm{L}$.

- $\quad \rho_{\text {comb }}$ - Densidad del combustible Fuel-Oil $=0,966$ kg/L [22].

5) Ahorro económico anual obtenido debido al aislamiento de las tuberías por donde circula vapor de agua con aislante Lana Mineral

Para determinar el ahorro económico obtenido a causa del aislamiento de las tuberías de vapor de agua con el aislante Lana Mineral se tomaron en cuenta tres pasos, los cuales se describen a continuación.

Paso 1. Determinación del ahorro energético alcanzado al aislar las tuberías:

$$
A_{\text {energ }}=P_{S A}-P_{C A}
$$

\section{Dónde:}

- $A_{\text {energ }}$ - Ahorro energético alcanzado al aislar las tuberías $[\mathrm{kJ} / \mathrm{h}]$.

- $\quad P_{S A}$ - Pérdidas de calor sin aislamiento $[\mathrm{kJ} / \mathrm{h}]$.

- $\quad P_{C A}$ - Pérdidas con aislamiento [kJ/h].

Paso 2. Cálculo del ahorro de vapor obtenido:

$$
A_{\text {vapor }}=\frac{A_{\text {energ }}}{h_{\text {vapor }}}
$$


Dónde:

- $A_{\text {vapor }}$ - Ahorro de vapor de agua obtenido producto al aislamiento de las tuberías $[\mathrm{kg} / \mathrm{h}]$.

- $\quad h_{\text {vapor }}$ - Entalpía del vapor de agua $=2767,50 \mathrm{~kJ} / \mathrm{kg}$ [23].

Paso 3. Cálculo del ahorro económico total anual obtenido a causa del aislamiento de las tuberías por donde circula vapor de agua:

Dónde:

- $A_{a i s l}$ - Ahorro económico total anual obtenido a causa del aislamiento de las tuberías [\$/año].

- $I_{v}^{m}$ - índice de generación de vapor de la caldera [ $\left.\mathrm{kg}_{\text {vapor }} / \mathrm{kg}_{\text {combustible }}\right]$ considerando las mejoras. Dato suministrado por la hoja de cálculo Excel utilizada para determinar la eficiencia energética del generador de vapor [17].

\section{6) Período de recuperación de la inversión}

Por último, el Período de Recuperación de la Inversión (PRI) se determinó a partir de la siguiente ecuación:[ST1]

$$
P R I=\frac{C_{i n v}^{t o t a l}}{A_{\text {precal }}+A_{\text {aisl }}}
$$

\section{RESULTADOS Y DISCUSIÓN}

A. Dureza total del agua de alimentación al generador de vapor

Se obtuvo una dureza total (DT) promedio de 20,52 ppm para el agua de alimentación al generador de vapor, la cual es 10,26 veces superior al valor establecido por norma según [11], que fija un límite máximo de $2 \mathrm{ppm}$. De esta manera se concluye que el sistema de ablandamiento de agua es inefectivo bajo las condiciones de operación actuales. Vale destacar que la dureza cálcica tuvo un valor promedio de 11,4 ppm, mientras que la dureza magnésica promedio fue de 9,12 ppm.

\section{B. Pérdidas de calor al ambiente y espesor óptimo del aislante}

La Tabla III muestra los resultados de las pérdidas de calor sin aislante y con aislante, el \% de reducción de las pérdidas de calor, asi como también el espesor y el diámetro del aislante obtenidos para los tramos de tubería horizontales, mientras que la Tabla IV expone los resultados de estos parámetros para las tuberías verticales.

TABLA III.

RESULTADOS OBTENIDOS DE PÉRDIDAS DE CALOR Y ESPESOR DEL AISLANTE PARA LAS TUBERÍAS HORIZONTALES.

\begin{tabular}{|c|c|c|c|c|c|}
\hline Tramo & $\begin{array}{c}\text { Pérdidas } \\
\text { de calor } \\
\text { ( } \sin \end{array}$ & $\begin{array}{l}\text { Pérdidas } \\
\text { de calor }\end{array}$ & $\begin{array}{l}\text { Reducción } \\
\text { de pérdidas }\end{array}$ & $\begin{array}{c}\text { Espesor } \\
\text { del }\end{array}$ & $\begin{array}{c}\text { Diámetro } \\
\text { del }\end{array}$ \\
\hline
\end{tabular}

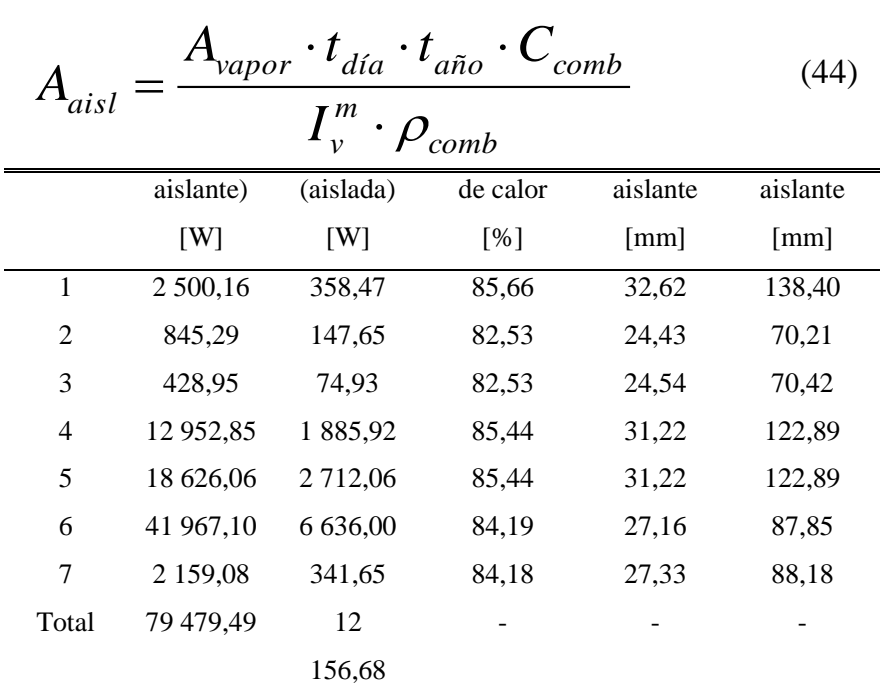

TABLA IV

RESULTADOS OBTENIDOS DE PÉRDIDAS DE CALOR Y ESPESOR DEL AISLANTE PARA LAS TUBERÍAS HORIZONTALES.

\begin{tabular}{|c|c|c|c|c|c|}
\hline Tramo & $\begin{array}{c}\text { Pérdidas } \\
\text { de calor } \\
\text { (sin } \\
\text { aislante) } \\
\text { [W] }\end{array}$ & $\begin{array}{c}\text { Pérdidas } \\
\text { de calor } \\
\text { (aislada) } \\
\text { [W] }\end{array}$ & $\begin{array}{c}\text { Reducción } \\
\text { de pérdidas } \\
\text { de calor } \\
\text { [\%] }\end{array}$ & $\begin{array}{c}\text { Espesor } \\
\text { del } \\
\text { aislante } \\
{[\mathrm{mm}]}\end{array}$ & $\begin{array}{c}\text { Diámetro } \\
\text { del } \\
\text { aislante } \\
{[\mathrm{mm}]}\end{array}$ \\
\hline 1 & 2238,39 & 235,34 & 88,49 & 38,79 & 150,73 \\
\hline 2 & 653,00 & 129,53 & 80,16 & 30,04 & 81,41 \\
\hline 3 & 384,33 & 56,96 & 85,18 & 26,03 & 81,34 \\
\hline 4 & 508,39 & 98,43 & 80,64 & 29,45 & 80,24 \\
\hline 5 & 1568,22 & 172,74 & 88,98 & 37,18 & 134,80 \\
\hline 6 & 2,08 & 0,08 & 96,04 & 34,40 & 129,36 \\
\hline 7 & 3,52 & 0,22 & 93,70 & 33,87 & 101,26 \\
\hline 8 & 926,89 & 113,09 & 87,80 & 29,34 & 93,63 \\
\hline Total & 6284,82 & 806,39 & - & - & - \\
\hline
\end{tabular}

Como bien se puede observar en las Tablas III y IV, las pérdidas totales para las tuberías horizontales sin aislar y aislada ascendió a $79479,49 \mathrm{~W}$ y $12156,68 \mathrm{~W}$, respectivamente, mientras que las pérdidas totales de las tuberías verticales sin aislar y aislada alcanzaron valores de $6284,82 \mathrm{~W}$ y 806,39 , respectivamente. Las pérdidas totales de las tuberías sin aislar, considerando las tuberías tanto horizontales como verticales, fueron de $85764,31 \mathrm{~W}$, mientras que las pérdidas totales de las tuberías aisladas tanto horizontales como verticales tuvieron un valor de 12 963,07 W. En los tramos horizontales se obtuvo un $\%$ de reducción de pérdidas de calor promedio de $84,28 \%$, 
mientras que en los tramos verticales el \% de reducción de pérdidas de calor promedio tuvo un valor de $87,62 \%$. El \% de reducción de pérdidas de calor total promedio, considerando las tuberías horizontales como verticales, fue de $85,95 \%$. El aislamiento de las tuberías por donde circula vapor de agua con el aislante Lana Mineral produce una reducción de las pérdidas de calor de $67322,81 \mathrm{~W}$ y $5478,43 \mathrm{~W}$ para los tramos horizontales y verticales, respectivamente. Se obtuvo una reducción de pérdidas de calor total de 72 801,24 W sumando tanto las tuberías horizontales como verticales. Por último, se obtuvo un espesor promedio de $28,36 \mathrm{~mm}$ y $32,38 \mathrm{~mm}$ para las tuberías horizontales y verticales, respectivamente, para un total promedio de $30,37 \mathrm{~mm}$.

\section{Eficiencia energética del generador de vapor}

En la Tabla V se desglosan los resultados de la eficiencia energética del generador de vapor, tanto para las condiciones actuales como considerando las mejoras, los cuales fueron obtenidos mediante la hoja de cálculo Excel utilizada para tal fin [16].

TABLA V.

RESULTADOS DE LA EFICIENCIA ENERGÉTICA DEL GENERADOR DE VAPOR.

\begin{tabular}{|c|c|c|c|}
\hline " Parámetro & $\begin{array}{c}\text { Valor } \\
\text { (sin } \\
\text { mejoras) }\end{array}$ & $\begin{array}{c}\text { Valor } \\
\text { (con } \\
\text { mejoras) }\end{array}$ & Ahorro \\
\hline
\end{tabular}

\begin{tabular}{cccc}
\hline Calor sensible de los gases de & 1173,92 & 1173,92 & - \\
combustión (kcal/kg) & & & - \\
Incombustión química (kcal/kg) & 9,99 & 9,99 & - \\
Incombustión mecánica (kcal/kg) & 39,47 & 39,47 & - \\
Radiación y convección (kcal/kg) & 19,02 & 8,88 & 10,14 \\
Factor de Evaporación & 1,1691 & 1,0951 & 0,074 \\
Índice de generación (kgvapor/kg & 13,663 & 14,603 & 0,94 \\
combustible) & & & \\
Eficiencia (\%) & 87,41 & 87,51 & 0,10 \\
Consumo de combustible (kg/h) & 365,95 & 342,39 & 23,56 \\
\hline
\end{tabular}

De forma general el generador de vapor está operando con una eficiencia energética de $87,41 \%$, la cual puede considerarse de aceptable [24] [25], mientras que el consumo de combustible es de $365,95 \mathrm{~kg} / \mathrm{h}$, el índice de generación es de 13,663 kg de vapor $/ \mathrm{kg}$ de combustible, y se obtuvo un factor de evaporación de 1,1691 para las condiciones actuales. La aplicación de las mejoras propuestas reduce el consumo de combustible (FuelOil) en $23,56 \mathrm{~kg} / \mathrm{h}$, incrementa la eficiencia energética del generador de vapor en $0,10 \%$ y aumenta el índice de generación en $0,94 \mathrm{~kg}$ de vapor $/ \mathrm{kg}$ de combustible, lo cual corrobora y ratifica la factibilidad técnica de las mejoras planteadas.

\section{Resultados de las emisiones de gases de combustión al ambiente}

La Tabla VI describe los resultados de las emisiones de los gases de combustión al ambiente, tanto para las condiciones actuales como considerando las mejoras.

TABLA VI

RESULTADOS DE LAS EMISIONES DE GASES DE COMBUSTIÓN AL AMBIENTE, TANTO PARA LAS CONDICIONES ACTUALES COMO CONSIDERANDO LAS MEJORAS.

\begin{tabular}{lcccccc}
\hline \hline Gas & & Emisiones (g/s) & & \multicolumn{3}{c}{ Emisones (kg/año) } \\
& Actual & Mejora & Ahorro & Actual & Mejora & Ahorro \\
\hline $\mathrm{CO}_{2}$ & 335,27 & 313,69 & 21,58 & 4098904,08 & 3835003,29 & 236900,79 \\
$\mathrm{~N}_{2}$ & 1487,20 & 1391,45 & 95,75 & 18181912,32 & 17011359,27 & 1170553,05 \\
$\mathrm{O}_{2}$ & 104,03 & 97,33 & 6,7 & 1271835,96 & 1189964,94 & 81871,02 \\
$\mathrm{SO}_{2}$ & 3,67 & 3,43 & 0,24 & 44826,52 & 41838,72 & 2987,80 \\
Total & 1930,17 & 1805,90 & 124,27 & 23597478,88 & 22078166,22 & 1492312,66 \\
\hline \hline
\end{tabular}

Como bien se puede observar en la Tabla VI, la introducción de las mejoras propuestas permite ahorrar anualmente 236 $900,79 \mathrm{~kg}$ de $\mathrm{CO}_{2}, 1170553,05 \mathrm{~kg}$ de $\mathrm{N}_{2}, 81871,02 \mathrm{~kg}$ de $\mathrm{O}_{2}$ y $2987,80 \mathrm{~kg}$ de $\mathrm{SO}_{2}$, para un ahorro total de emisiones de 1 $492312,66 \mathrm{~kg} / \mathrm{año}$, el cual puede considerarse de significativo e importante.

Según la hoja de cálculo Excel utilizada para determinar la eficiencia energética del generador de vapor [17], la composición de los gases de combustión producidos en el generador de vapor alcanzó los valores porcentuales que se muestran en la Tabla VII.

TABLA VII.

COMPOSICIÓN PORCENTUAL DE LOS GASES DE COMBUSTIÓN PRODUCIDOS DURANTE LA GENERACIÓN DE VAPOR.

Gases $\%$ en peso $\left(\mathrm{x}_{\mathrm{i}}\right) \quad \%$ en volumen $\left(\mathrm{y}_{\mathrm{i}}\right)$

\begin{tabular}{ccc}
\hline $\mathrm{CO}_{2}$ & 17,37 & 11,83 \\
$\mathrm{~N}_{2}$ & 77,05 & 83,00 \\
$\mathrm{O}_{2}$ & 5,39 & 5,08 \\
$\mathrm{SO}_{2}$ & 0,19 & 0,09 \\
\hline
\end{tabular}

La determinación del flujo volumétrico de la mezcla de gases de combustión mediante (28) y (29) permitió conocer que, en las condiciones actuales, se emiten $2,37 \mathrm{~m}^{3} / \mathrm{s}$, mientras que con la aplicación de las mejoras planificadas se emitirán $2,21 \mathrm{~m}^{3} / \mathrm{s}$, esto es, se obtendrá una reducción en la emisión de los gases de combustión de $0,16 \mathrm{~m}^{3} / \mathrm{s}\left(576 \mathrm{~m}^{3} / \mathrm{h}\right)$.

La Fig. 3 muestra los resultados obtenidos mediante el software Contamin, con respecto a la estimación de la dispersión de los gases contaminantes, tanto para las condiciones actuales como las mejoras propuestas. 


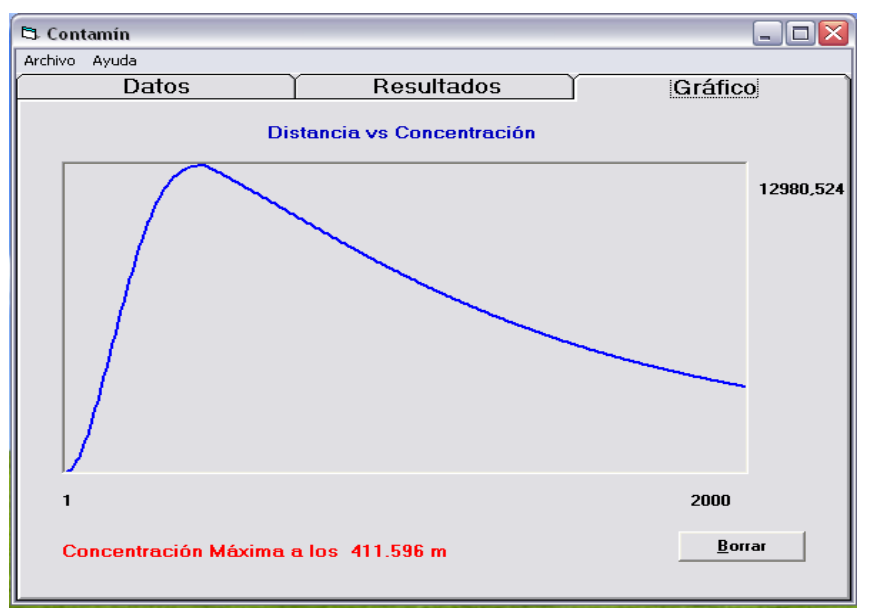

a)

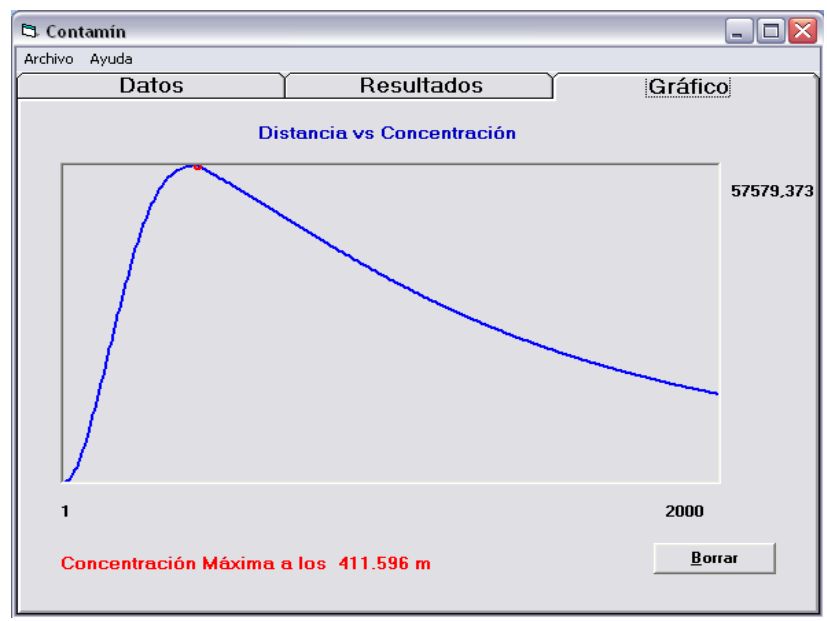

c)

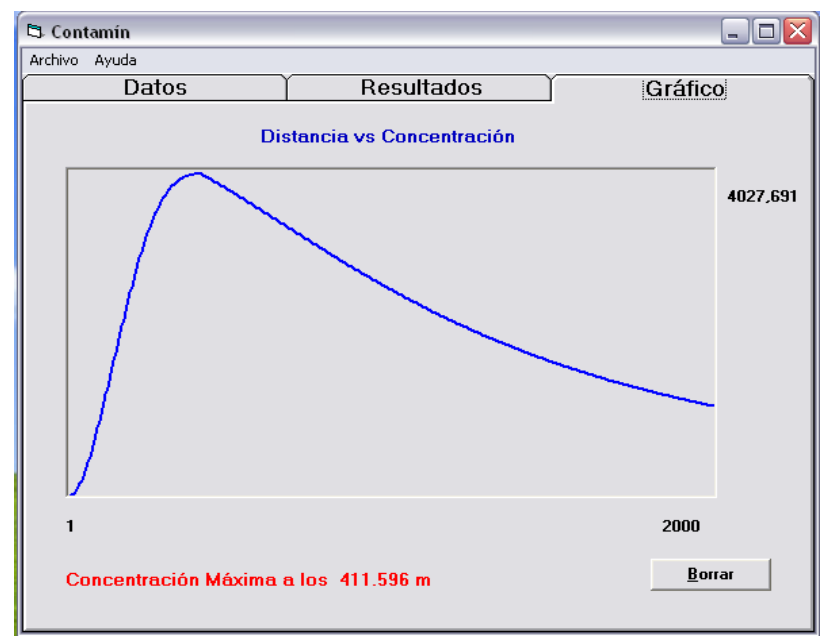

e)

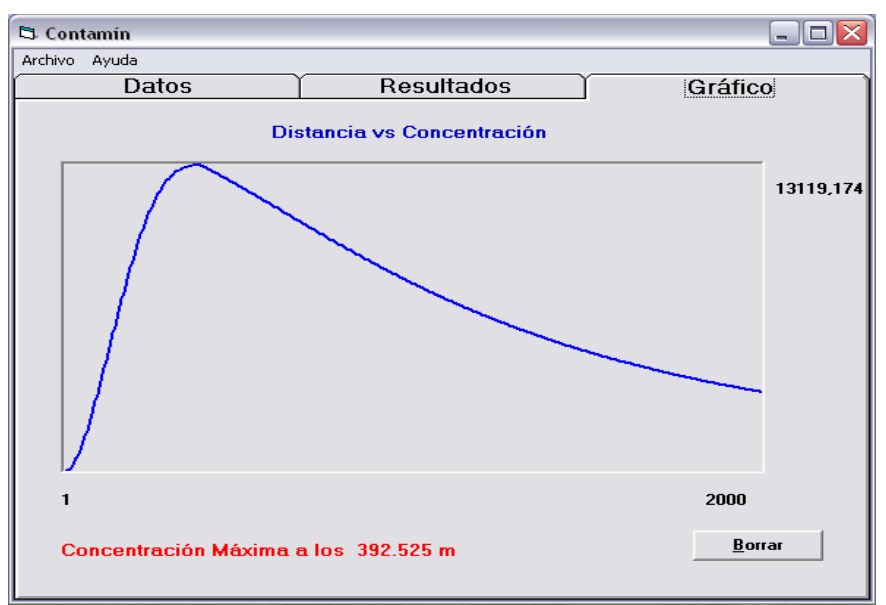

b)

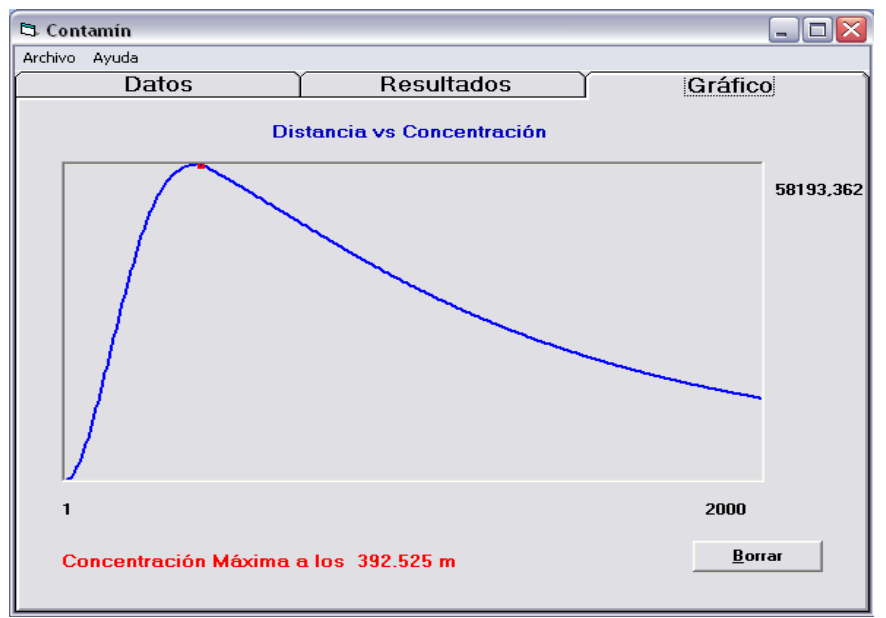

d)

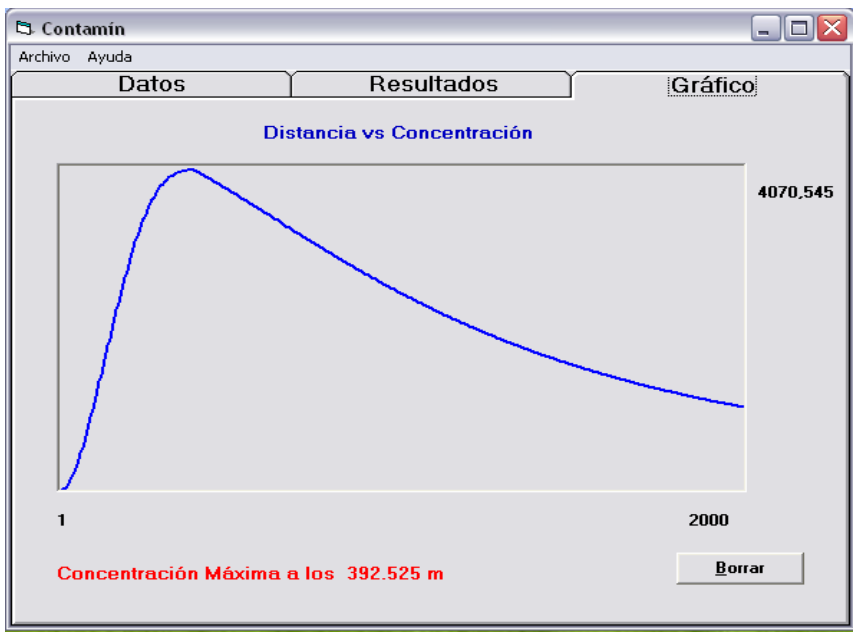

f) 


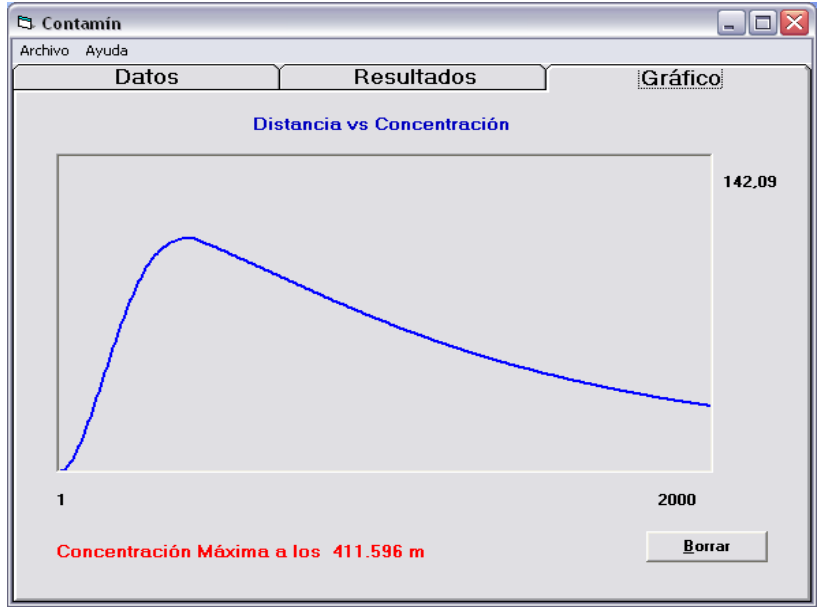

g)

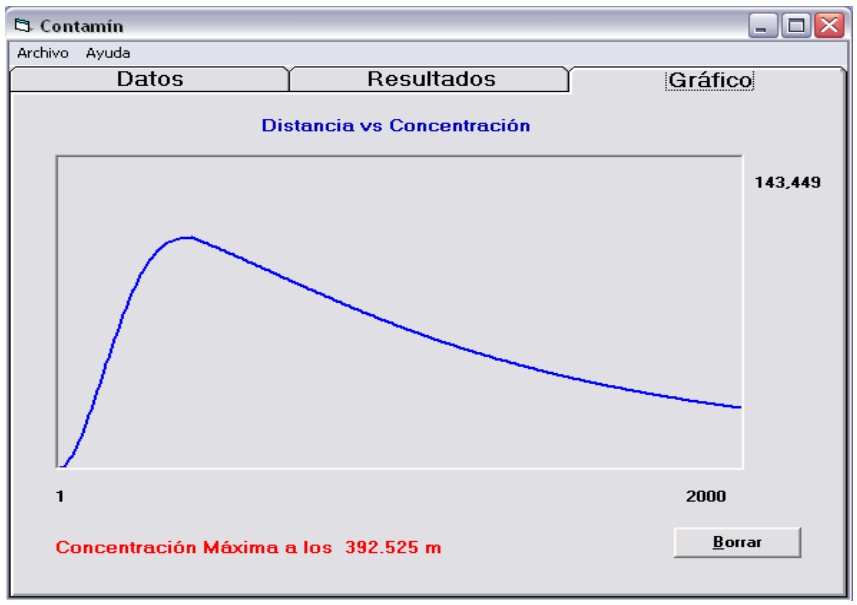

h)

Fig. 3. Resultados de la dispersión de los gases de combustión obtenidos mediante el software Contamin.

a) $\mathrm{CO}_{2}$ sin considerar las mejoras.

b) $\mathrm{CO}_{2}$ considerando las mejoras.

c) $\mathrm{N}_{2} \sin$ considerar las mejoras.

d) $\mathrm{N}_{2}$ considerando las mejoras.

e) $\mathrm{O}_{2}$ sin considerar las mejoras.

f) $\mathrm{O}_{2}$ considerando las mejoras.

g) $\mathrm{SO}_{2}$ sin considerar las mejoras.

h) $\mathrm{SO}_{2}$ considerando las mejoras.

Considerando los resultados mostrados en la Fig. 3, se puede establecer que se alcanza una concentración máxima de los gases de combustión a los 411,596 m y 392,525 m del punto emisor para las condiciones actuales y para las alternativas de mejoras, respectivamente. $\mathrm{El} \mathrm{CO}_{2}$ alcanza una concentración máxima de $12980,524 \mu \mathrm{g} / \mathrm{m}^{3}$ para las condiciones actuales, y de $13119,174 \mu \mathrm{g} / \mathrm{m}^{3}$ para las alternativas de mejoras. Por su parte, el $\mathrm{N}_{2}, \mathrm{O}_{2}$ y SO $\mathrm{S}_{2}$ alcanzan concentraciones máximas de 57 $579,373 \mu \mathrm{g} / \mathrm{m}^{3}, 4027,691 \mu \mathrm{g} / \mathrm{m}^{3}$ y $142,090 \mu \mathrm{g} / \mathrm{m}^{3}$ para las condiciones actuales, y de $58193,362 \mu \mathrm{g} / \mathrm{m}^{3}, 4070,545 \mu \mathrm{g} / \mathrm{m}^{3}$ y $143,449 \mu \mathrm{g} / \mathrm{m}^{3}$ considerando las alternativas de mejoras, respectivamente.

\section{E. Resultados de los cálculos económicos}

1) Costo de inversión del aislamiento de las tuberías por donde circula vapor de agua

La siguiente tabla (Tabla VIII) desglosa los costos relacionados con el aislamiento de cada uno de los tramos de tuberías por donde circula vapor de agua, en dependencia del diámetro nominal de las tuberías y de la longitud total de estas. También muestra el costo de adquisición total que deberá invertirse para adquirir el material aislante necesario para aislar todos los tramos de tuberías.

TABLA VIII.

COSTO TOTAL DE ADQUISICIÓN DEL MATERIAL AISLANTE LANA MINERAL CON RECUBRIMIENTO DE ALUMINIO, NECESARIO PARA AISLAR TODOS LOS

\begin{tabular}{ccccc}
\multicolumn{5}{c}{ TRAMOS DE TUBERÍAS. } \\
\hline $\begin{array}{c}\text { Diámetro } \\
\text { nominal } \\
{[\mathrm{pulg}]}\end{array}$ & $\begin{array}{c}\text { Espesor del } \\
\text { aislante } \\
{[\mathrm{mm}]}\end{array}$ & $\begin{array}{c}\text { Longitud } \\
\text { total tubería } \\
{[\mathrm{m}]}\end{array}$ & $\begin{array}{c}\text { Precio } \\
\text { unitario } \\
{[\mathrm{PC} \$ / \mathrm{m}]}\end{array}$ & $\begin{array}{c}\text { Costo de } \\
\text { adquisición } \\
{[\mathrm{PC} \$]}\end{array}$ \\
\hline $1 / 2$ & 25 & 23,25 & 3,58 & 83,235 \\
1 & 25 & 257,25 & 6,8 & 1749,300 \\
$21 / 2$ & 40 & 13,5 & 7,27 & 98,145
\end{tabular}

\begin{tabular}{cllll}
2 & 40 & 117,2 & 20,06 & 2351,032 \\
Total & 411,2 & & 4281,712 \\
\hline
\end{tabular}

Luego, el costo total de inversión del material aislante, considerando además el costo de instalación como un $2 \%$ del costo total de adquisición, será determinado mediante (39). De esta manera:

$$
\begin{aligned}
& C_{i n v}^{a i s l}=C_{a i s l}+C_{a i s l} \cdot 0,02 \\
& C_{i n v}^{a i s l}=\text { PC } \$ 4367,35
\end{aligned}
$$

Se requieren PC \$ 4 367,35 para aislar los 411,2 m de tramos de tuberías por donde fluye vapor de agua con el aislante Lana Mineral y recubrimiento de aluminio, considerando tanto la adquisición del material aislante como su instalación.

\section{2) Costo de inversión total}

Tomando en cuenta que el costo total de adquisición del sistema de precalentamiento del agua de alimentación al generador de vapor es de PC \$3750, entonces el costo de inversión total relacionado con la instalación y montaje tanto del material de aislamiento de las tuberías por donde fluye vapor de agua como el sistema de precalentamiento del agua de alimentación a la caldera, se determinó mediante (40), Asi:

$$
\begin{gathered}
C_{i n v}^{\text {total }}=C_{i n v}^{a i s l}+C_{i n v}^{\text {precal }} \\
C_{i n v}^{\text {total }}=\text { PC } \$ 8117,35 .
\end{gathered}
$$

En resumen, se deben invertir PC \$ 8 117,35 para implementar ambas alternativas de mejoras propuestas. 
3) Ahorro económico anual obtenido a causa de la implementación del sistema de precalentamiento del agua de alimentación la generador de vapor

Para determinar cuánto se ahorra anualmente debido a la implementación del sistema de precalentamiento del agua de alimentación al generador de vapor se recurrió a (41). Entonces:

$$
A_{\text {precal }}=\frac{A_{c o m b} \cdot t_{\text {día }} \cdot t_{\text {año }} \cdot C_{c o m b}}{\rho_{c o m b}}
$$

Dónde:

$$
\begin{array}{ll}
\text { - } & A_{\text {comb }}=23,56 \mathrm{~kg} / \mathrm{h}(\text { Tabla V }) . \\
\text { - } & t_{\text {día }}=12 \mathrm{~h} / \mathrm{día} . \\
\text { - } & t_{\text {año }}=283 \text { día/año. } \\
\text { - } & C_{c o m b}=\text { PC } \$ 0,39 / \mathrm{L} . \\
\text { - } & \rho_{\text {comb }}=0,966 \mathrm{~kg} / \mathrm{L}[22] .
\end{array}
$$

Seguidamente, sustituyendo estos valores en (41) se tiene:

$$
A_{\text {precal }}=\text { PC } \$ 32302,08 / \text { año }
$$

La implementación del sistema de precalentamiento del agua de alimentación a la caldera permitirá un ahorro económico anual de PC \$ 32 302,08 debido a combustible que no se consume en este equipo, el cual puede considerarse de significativo.

4) Ahorro económico anual obtenido a causa del aislamiento de las tuberías por donde circula vapor de agua con aislante Lana Mineral

Se seguirá la metodología de cálculo descrita en el Capítulo 2 a través de los tres pasos descritos en esta. Así:

Paso 1. Ahorro energético alcanzado al aislar las tuberías. Se hizo uso de (42):

$$
A_{\text {energ }}=P_{S A}-P_{C A}
$$

Dónde:

$$
\begin{aligned}
& P_{S A}=85764,31 \mathrm{~W}=308753,23 \mathrm{~kJ} / \mathrm{h} . \\
& P_{C A}=12963,07 \mathrm{~W}=46667,31 \mathrm{~kJ} / \mathrm{h} .
\end{aligned}
$$

Entonces se tiene que:

$$
A_{e}
$$$$
{ }_{\text {energ }}=262085,92 \mathrm{~kJ} / \mathrm{h} \text {. }
$$

Paso 2. Ahorro de vapor de agua. Se empleó (43):

$$
A_{\text {vapor }}=\frac{A_{\text {energ }}}{h_{\text {vapor }}}
$$

Dónde:

$$
\text { - } \quad h_{\text {vapor }}=2767,50 \mathrm{~kJ} / \mathrm{kg}[23] \text {. }
$$

Luego se obtiene:

$$
\text { A }
$$$$
A_{\text {vapor }}=94,70 \mathrm{~kg} / \mathrm{h} \text {. }
$$

Paso 3. Ahorro económico total anual obtenido a causa del aislamiento de las tuberías por donde circula vapor de agua.

Para determinar este parámetro se utilizó (44).

$$
A_{\text {aisl }}=\frac{A_{\text {vapor }} \cdot t_{\text {dia }} \cdot t_{\text {año }} \cdot C_{c o m b}}{I_{v}^{m} \cdot \rho_{c o m b}}
$$

Dónde:

- $\quad I_{v}^{m}=14,603 \mathrm{~kg}_{\text {vapor }} / \mathrm{kg}_{\text {combustible }}$ (Tabla V)

- $t_{\text {día }}=12 \mathrm{~h} /$ día

- $\quad t_{\text {año }}=283$ día/año.

- $C_{\text {comb }}=$ PC $\$ 0,39 / \mathrm{L}$.

- $\quad \rho_{\text {comb }}=0,966 \mathrm{~kg} / \mathrm{L}[22]$.

Sustituyendo estos datos en (44) se obtiene: $A_{\text {aisl }}=$ PC $\$ 8$ 891,25/año.

El ahorro económico total anual que se obtiene debido al aislamiento de las tuberías por donde circula vapor con el aislante Lana Mineral ascendió a PC \$ 8 891,25, el cual se debe fundamentalmente a un incremento del índice de generación de vapor de la caldera hasta alcanzar un valor de 14,603

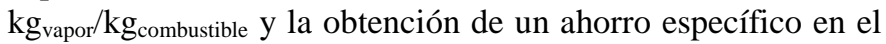
consumo de vapor $(94,70 \mathrm{~kg} / \mathrm{h})$.

\section{5) Periodo de recuperación de la inversión}

A continuación se determinó el Período de Recuperación de la Inversión (PRI) mediante (45):

$$
P R I=\frac{C_{\text {inv }}^{\text {total }}}{A_{\text {precal }}+A_{\text {aisl }}}
$$

Dónde:

$$
\begin{array}{ll}
\text { - } & C_{i n v}^{\text {total }}=\text { PC } \$ 8117,35 \\
\text { - } & A_{\text {precal }}=\text { PC } \$ 32 \text { 302,08/año } \\
\text { - } & A_{\text {aisl }}=\text { PC } \$ 8 \text { 891,25/año. }
\end{array}
$$

De esta manera, sustituyendo estos valores en (45) se tiene:

$P R I=0,197$ años $=2,36$ meses.

El costo total de inversión necesario para implementas ambas propuestas de mejoras alcanzó un valor de PC \$ 8 117,346, el cual se recupera en 2,36 meses ( 0,197 años) tomando en cuenta el ahorro económico total anual que se obtiene (PC \$ 41 193,33), el cual se debe fundamentalmente al ahorro en la producción de vapor $(94,70 \mathrm{~kg} / \mathrm{h})$ y en el consumo de combustible en la caldera $(23,56 \mathrm{~kg} / \mathrm{h})$, así como también al incremento del índice de generación de vapor de la caldera en $0,94 \mathrm{~kg}_{\text {vapor }} / \mathrm{kg}_{\text {combustible. }}$

\section{CONCLUSIONES}

El agua de alimentación a la caldera presenta una dureza total promedio de 20,52 ppm, la cual es 10,26 veces superior al valor establecido por norma ( $2 \mathrm{ppm})$, por lo que se establece que el sistema de ablandamiento de esta agua de la instalación es 
inefectivo.

El aislamiento de las tuberías por donde circula vapor de agua con el aislante Lana Mineral permite reducir las pérdidas de calor al ambiente en $72801,24 \mathrm{~W}$.

La caldera está operando con una eficiencia energética de $87,41 \%$, la cual se considera de aceptable.

La aplicación de las alternativas de mejoras propuestas reduce el consumo de combustible en $23,56 \mathrm{~kg} / \mathrm{h}$, incrementa la eficiencia energética en $0,10 \%$ y aumenta el índice de generación en $0,94 \mathrm{~kg}$ de vapor $/ \mathrm{kg}$ de combustible del generador de vapor.

La aplicación de las alternativas de mejoras propuestas permite ahorrar anualmente la emisión al ambiente de 236 $900,79 \mathrm{~kg}$ de $\mathrm{CO}_{2}, 1170553,05 \mathrm{~kg}$ de $\mathrm{N}_{2}, 81871,02 \mathrm{~kg} \mathrm{de} \mathrm{O}_{2}$ y $2987,80 \mathrm{~kg}$ de $\mathrm{SO}_{2}$, para un ahorro total de emisiones de 1 $492312,66 \mathrm{~kg} /$ año.

Se alcanza una concentración máxima de los gases de combustión a los 411,596 m y 392,525 m del punto emisor para las condiciones actuales y para las alternativas de mejoras, respectivamente.

La implementación de ambas alternativas de mejoras propuestas requiere una inversión total de capital de PC \$8 117,35 , la cual se recupera en 2,36 meses considerando el ahorro económico total anual que se obtiene, que asciende a PC \$ 41193,33 .

\section{REFERENCIAS}

[1] M. J. Lapido, D. A. Vidal, y J. A. Madrigal, Funcionamiento y pérdidas en calderas pirotubulares. Estudios de casos, Cienfuegos, Cuba: Editorial "Universo Sur", 2015, pp. 12-27.

[2] ATECYR. Guía técnica sobre procedimiento de inspección periódica de eficiencia energética para calderas, Madrid, España: Instituto para la Diversificación y Ahorro de la Energía (IDAE), 2007, pp. 8-17.

[3] K. S. Venkateswarlu, Water Chemistry. Industrial and Power Station Water Treatment. New Delhi, India: New Age International (P) Ltd., Publishers, 1996, pp. 32-48.

[4] M. A. Golato, F. J. F. Colombres, G. Aso, C. A. Correa, y D. Paz, "Metodología de cálculo de la eficiencia térmica de generadores de vapor," Rev. Ind. y Agríc. de Tucumán, vol. 85, no. 2, pp. 17-31. Dic. 2008.

[5] R. Jiménez, M. J. Lapido, O. Herrera, y E. Cabezas, "Influencia de los parámetros operacionales en la eficiencia termodinámica de instalaciones de baja y mediana capacidad," Centro Azúcar, vol. 42, no. 3, pp. 8088, Jul.-Sep. 2015.

[6] R. Jiménez, M. J. Lapido, J. A. Madrigal, y D. A. Vidal, "Método para la evaluación de la eficiencia e impacto ambiental de un generador de vapor," Ingeniería Energética, vol. XXXVII, no. 2, pp. 135-144. MayoAgosto 2016.

[7] R. F. Güiza, y V. H. Rangel, "Metodología para determinar la eficiencia energética de calderas de baja potencia," Verano de la Investigación Científica, vol. 3, no. 2, pp. 2628-2634, 2017.
[8] M. A. Aguilar, "Estudio de la eficiencia energética y de combustión de una caldera utilizada en una planta de fabricación de alimentos y propuesta para incrementar la eficiencia de operación," Tesis de Diploma, Facultad de Ingeniería, Universidad San Carlos de Guatemala, Guatemala, 2015.

[9] S. Krishnanunni, P. C. Josephkunju, M. Potti, and E. M. Mathew, "Evaluation of Heat Losses in Fire Tube Boiler," International Journal of Emerging Technology and Advanced Engineering, vol. 32, no. 12, pp. 301-305. Dec. 2012.

[10] Boiler Water Quality Requirements and Associated Steam Quality for Industrial/Commercial and Institutional Boilers. American Boiler Manufacturers Association (ABMA), 2005.

[11] Recommendations for treatment of water for steam boilers and water heaters, British Standard 2486:1997, 1997.

[12] Feed Water, Boiler Water and Steam Quality for Power Plants/Industrial Plants, VGB-S-010-T-00;2011-12.EN, 2011.

[13] Calidad del agua - Determinación de la suma de calcio y magnesio - Método por valoración con EDTA (ISO 6059:1984, IDT), NC ISO 6059, 2010.

[14] Calidad del agua - Determinación del contenido de calcio - Método por valoración con EDTA (ISO 6058: 1984, IDT), NC ISO 6058, 2009.

[15] A. Pérez, "Cálculo de las pérdidas de calor y el espesor óptimo del aislante para tuberías tanto horizontales como verticales," Universidad de Camagüey, Versión 1.3, 2013.

[16] A. Fonte, P. Nemirovich y R. González, Cálculo de la pérdidas de calor y del espesor del aislante, Camagüey, Cuba: Facultad de Ingeniería Química, Universidad de Camagüey, 1986, pp. 6-12.

[17] P. Rodríguez, "Sistema de Análisis de Eficiencia para Generadores de Vapor," MEP, Version 2.0, 2006.

[18] N. Tanquero, D. Martínez, V. Castillo y G. Fernández, Curso de transporte del vapor en la industria, La Habana, Cuba: Ingeniería Alastor, 2010, pp. 12-27.

[19] CUJAE, "Contamin," Universidad Tecnológica de La Habana Jose Antonio Hecheverria, Versión 1.0.0, 2004.

[20] K. F. Pávlov, P. G. Romankov y A. A. Noskov, Problemas y ejemplos para el curso de operaciones básicas y aparatos en tecnología química, Moscú, Rusia: Editorial Mir, 1981, pp. 24-26.

[21] M. S. Peters, K. D. Timmerhaus and R. E. West, Plant Design and Economics for Chemical Engineers, New York, USA: McGraw-Hill, 2003, pp. 128-133.

[22] GPSA, Engineering Data Book, 12th ed. Tulsa, USA: Gas Processors Suppliers Association, 2004, pp. 23-2 - 23-8.

[23] ChemicaLogic Corporation, "Thermodynamic and Transport Properties of Water and Steam," ChemicaLogic $^{\circledR}$ Steam Tab Companion, Version 2.0, 2003.

[24] V. Ganapathy, Industrial Boilers and Heat Recovery Steam Generators Design, Applications, and Calculations, New York, USA: Marcel Dekker, 2003, pp. 68-72.

[25] K. E. Heselton, Boiler Operator's Handbook, Lilburn, USA: Fairmont Press, Inc, 2005, pp. 105-112. 


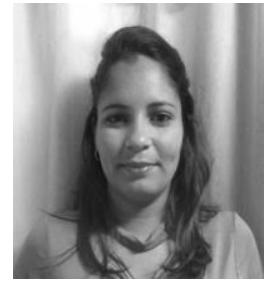

Yasneli Reyes Betancourt nació en Camagüey, Cuba en 1994. Recibió el título de Ingeniero Químico en la Universidad de Camagüey, Cuba en el año 2019. Actualmente se desempeña profesionalmente como Especialista de Transportación Ferroviaria en el Departamento de Material Rodante y Talleres de la Empresa de Ferrocarriles Centro-Este, de Camagüey, Cuba. Su área de investigación abarca la evaluación termodinámica de sistemas y procesos de la industria química, con énfasis en la industria cárnica.

ORCID: https://orcid.org/0000-0002-3334-0381.

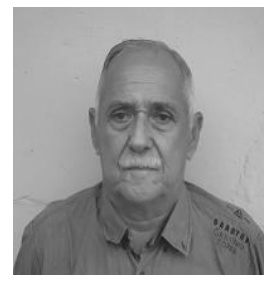

Raúl González de la Cruz nació en Camagüey, Cuba en 1944. Recibió el título de Ingeniero Químico en la Universidad de Camagüey, Cuba en el año 1978. Se desempeña profesionalmente como Profesor Auxiliar Consultante en la Universidad de Camagüey, Cuba. Su área de investigación comprende la evaluación termo-energética y termodinámica de sistemas, operaciones y procesos de la industria química, cálculos medioambientales y diseño de equipos de transferencia de calor y masa. Es Máster en Análisis de Procesos de la Industria Química desde el año 2008.

ORCID: https://orcid.org/0000-0002-2621-239X.

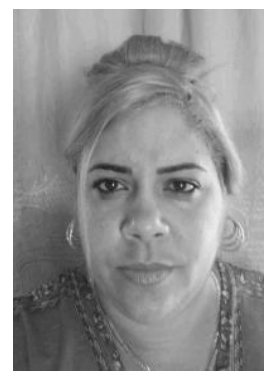

Yilena Bárbara Rodríguez Guerra nació en Camagüey, Cuba, en el año 1983. Recibió el título de Ingeniero Químico en la Universidad de Camagüey en el año 2009. Se desempeña profesionalmente como Especialista del área de producción del Combinado Cárnico de Camagüey. Su área de investigación comprende la evaluación termoenergética de sistemas de generación y distribución de vapor de agua, así como también el control de calidad de productos y elaboraciones de la industria cárnica.

ORCID: https://orcid.org/0000-0002-1059-6253.

Norlem Liaño Abascal nació en Camagüey, Cuba en 1984. Recibió el título de Ingeniero Químico en la Universidad de Camagüey, Cuba en el año 2007. Se desempeña profesionalmente como Profesor Asistente en la Universidad de Camagüey, Cuba. Su área de investigación abarca la evaluación termodinámica técnica de procesos y operaciones de la industria química y energética, el diseño de equipos de

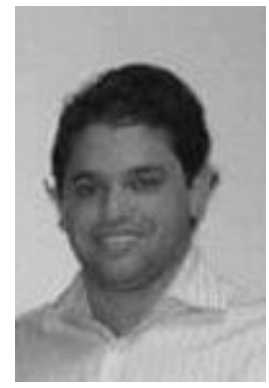
transferencia de calor y masa, y el empleo de simuladores para la evaluación técnicoeconómica de plantas químicas. Es Máster en Análisis de Procesos en la Industria Química desde el año 2012.

ORCID: https://orcid.org/0000-00018032-0423.

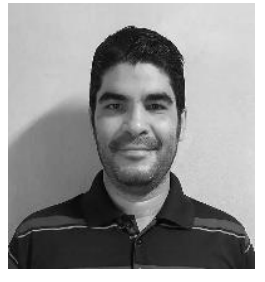

Amaury Pérez Sánchez nació en Camagüey, Cuba en 1984. Recibió su título de Ingeniero Químico por parte de la Universidad de Camagüey en 2009. Actualmente se desempeña como profesor instructor de la Facultad de Ciencias Aplicadas, Universidad de Camagüey. Sus áreas de investigación incluyen la modelación y simulación de procesos químicos y biotecnológicos, diseño de equipos de transferencia de calor y masa, y el diseño de plantas y operaciones de la industria biotecnológica.

Su producción abarca la publicación de 30 artículos en revistas científicas, y la elaboración de un libro de simulación de procesos. Actualmente se encuentra cursando una Maestría en Biotecnología.

ORCID: http://orcid.org/0000-0002-0819-6760. 